\title{
Bare Lymphocyte Syndrome
}

National Cancer Institute

\section{Source}

National Cancer Institute. Bare Lymphocyte Syndrome. NCI Thesaurus. Code C3895.

A hereditary immunodeficiency disorder caused by the absence of major

histocompatibility complex class II expression. Signs include upper and lower respiratory

tract bacterial infections, malabsorption, diarrhea, and mucocutaneous candidiasis. 